\title{
ON THE VERTEX-FACE GRAPHS OF TRIANGULATIONS*
}

\author{
WEIGEN YAN ${ }^{\dagger}$ AND SHULI LI ${ }^{\ddagger}$
}

\begin{abstract}
Let $G=(V(G), E(G))$ be a triangulation with vertex set $V(G)=\left\{v_{1}, v_{2}, \ldots, v_{n}\right\}$ and edge set $E(G)$ embedded on an orientable surface with genus $g$. Define $G^{\nabla}$ to be the graph obtained from $G$ by inserting a new vertex $v_{\phi}$ to each face $\phi$ of $G$ and adding three new edges $\left(u, v_{\phi}\right),\left(v, v_{\phi}\right)$ and $\left(w, v_{\phi}\right)$, where $u, v$ and $w$ are the three vertices on the boundary of $\phi$. Let $G^{\square}$ be the graph obtained from $G^{\nabla}$ by deleting all edges in $E(G)$ of $G^{\nabla}$. In this paper, first some spectral properties of $G^{\nabla}$ and $G^{\square}$ are considered, then it is proved that $t\left(G^{\nabla}\right)=3^{n+4 g-3} 5^{n-1} t(G)$ and $t\left(G^{\square}\right)=3^{n+4 g-3} 2^{n-1} t(G)$, where $t(G)$ is the number of spanning trees of $G$. As applications, the number of spanning trees and Kirchhoff indices of some lattices in the context of statistical physics are obtained.
\end{abstract}

Key words. Vertex-face graph, Triangulation, Spanning tree, Kirchhoff index, Degree Kirchhoff index.

AMS subject classifications. 05C30, 05C50, 05C76, 05C90.

1. Introduction. Unless otherwise specified, graphs in this paper are simple. Let $A_{G}$ and $D_{G}$ denote the adjacency matrix and the diagonal matrix of vertex degrees of the graph $G$ with $n$ vertices, respectively. The matrices $L_{G}=D_{G}-A_{G}$ and $\mathcal{L}_{G}=D_{G}^{-1 / 2} L_{G} D_{G}^{-1 / 2}$ are called the Laplacian matrix and the normalized Laplacian matrix of $G$, respectively. The polynomials $\mu(G, x)=\operatorname{det}\left(x I_{n}-L_{G}\right)$ and $\chi(G, x)=\operatorname{det}\left(x I_{n}-\mathcal{L}_{G}\right)$ are called the Laplacian characteristic polynomial and the normalized Laplacian characteristic polynomial of $G$, respectively.

Let $G$ be a triangulation embedded on an orientable surface $\Sigma$ with genus $g$ with vertex set $V(G)=$ $\left\{v_{1}, v_{2}, \ldots, v_{n}\right\}$, edge set $E(G)=\left\{e_{1}, e_{2}, \ldots, e_{m}\right\}$, and face set $F(G)=\left\{\phi_{1}, \phi_{2}, \ldots, \phi_{f}\right\}$. The dual graph $G^{\perp}$ of $G$ is the graph whose vertices, edges and faces correspond to faces, edges and vertices of $G$, respectively. We can embed $G$ and $G^{\perp}$ simultaneously in $\Sigma$ such that an edge $e$ of $G$ crosses the corresponding dual edge $e^{\perp}$ of $G^{\perp}$ exactly once and crosses no other edge of $G^{\perp}$. Hence, $V\left(G^{\perp}\right)=F(G), E\left(G^{\perp}\right)=\left\{e_{1}^{\perp}, e_{2}^{\perp}, \ldots, e_{m}^{\perp}\right\}$, and $F\left(G^{\perp}\right)=V(G)$.

By the Eulerian formula, $n-m+f=2-2 g$. Let $V(\phi)$ denote the set of vertices on the boundary of face $\phi$ of $G$. Note that each face $\phi$ of $G$ (including the unbounded face) is a triangle. So $|V(\phi)|=3$ and $2 m=3 f$. Hence, $f=2 n+4 g-4$ and $m=3 n+6 g-6$. Now we define two new graphs $G^{\nabla}$ and $G^{\square}$ from $G$, which are called the vertex-face graph of the first kind and the second kind of $G$, respectively, as follows. The vertex sets $V\left(G^{\nabla}\right)$ and $V\left(G^{\square}\right)$ of $G^{\nabla}$ and $G^{\square}$ are the union of $V(G)$ and $F(G)$, i.e., $V\left(G^{\nabla}\right)=V\left(G^{\square}\right)=V(G) \cup F(G)$, and the edge sets $E\left(G^{\nabla}\right)=E(G) \cup\{(u, \phi) \mid u \in V(\phi), \phi \in F(G)\}$ and $E\left(G^{\square}\right)=\{(u, \phi) \mid u \in V(\phi), \phi \in F(G)\}$. Obviously, $G^{\nabla}$ is a triangulation with $n+f$ vertices embedded on $\Sigma$, and $G^{\square}$ is a bipartite graph with $n+f$ vertices and $m$ faces embedded on $\Sigma$. Furthermore, $|V(\phi)|=4$ for every face $\phi \in F\left(G^{\square}\right)$. If $G$ is the complete graph with four vertices which is embedded on the plane as illustrated in Figure 1(a), then the corresponding vertex-face graphs $G^{\nabla}$ and $G^{\square}$ are the graphs illustrated in Figure 1(b) and (c), respectively.

* Received by the editors on July 23, 2018. Accepted for publication on July 20, 2020. Handling Editor: Bryan L. Shader. Corresponding Author: Shuli Li.

†School of Sciences, Jimei University, Xiamen 361021, China (weigenyan@263.net).

${ }^{\ddagger}$ School of Mathematical and Computer Sciences, Quanzhou Normal University, Quanzhou 362000, China (lishuli198710@163.com). 


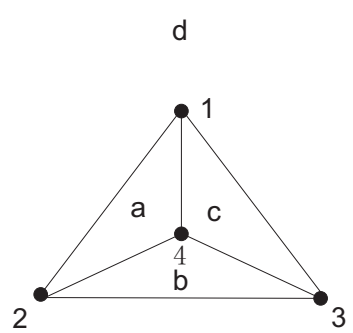

(a)

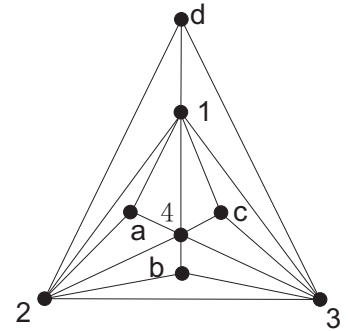

(b)

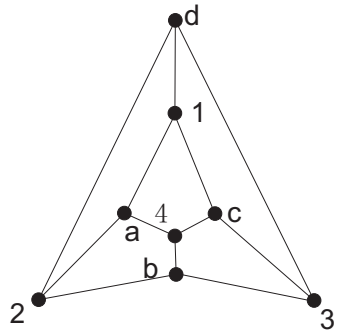

(c)

Figure 1. (a) A plane triangulation $G$ with vertex set $V(G)=\{1,2,3,4\}$ and face set $F(G)=\{a, b, c, d\}$. (b) The vertex-face graph $G^{\nabla}$ of the first kind of $G$. (c) The vertex-face graph $G^{\square}$ of the second kind of $G$.

In this paper, we first consider some spectral properties of $G^{\nabla}$ and $G^{\square}$ in Section 2. In Section 3, we prove that $t\left(G^{\nabla}\right)=3^{n+4 g-3} 5^{n-1} t(G)$ and $t\left(G^{\square}\right)=3^{n+4 g-3} 2^{n-1} t(G)$, where $t(G)$ is the number of spanning trees of $G$, which is also referred to as the complexity of $G$. In Section 4, we obtain a relation between $\operatorname{Kf}\left(G^{\nabla}\right), \operatorname{Kf}\left(G^{\square}\right)$ and $\operatorname{Kf}(G)$ and also obtain a relation between $\operatorname{Kf}^{\prime}\left(G^{\nabla}\right), \mathrm{Kf}^{\prime}\left(G^{\square}\right)$ and $\operatorname{Kf}^{\prime}(G)$, where $\operatorname{Kf}^{\square}(G)$ and $\mathrm{Kf}^{\prime}(G)$ are the Kirchhoff index and degree-Kirchhoff index of $G$, respectively. As applications, in Section 5 , we compute the number of spanning trees and Kirchhoff indices of some lattices in the context of statistical physics.

2. Spectral properties of $G^{\nabla}$ and $G^{\square}$. Suppose that $G$ is a triangulation embedded on an orientable surface with vertex set $V(G)=\left\{v_{1}, v_{2}, \ldots, v_{n}\right\}$, edge set $E(G)=\left\{e_{1}, e_{2}, \ldots, e_{m}\right\}$, and face set $F(G)=$ $\left\{\phi_{1}, \phi_{2}, \ldots, \phi_{f}\right\}$. Define the vertex-face incident matrix as $M_{G}=\left\{m_{i j}\right\}_{n \times f}$, where $m_{i j}=1$ if vertex $v_{i} \in V\left(\phi_{j}\right)$ and $m_{i j}=0$ otherwise. For the triangulation $G$ illustrated in Figure $1(\mathrm{a}), V(G)=\{1,2,3,4\}$ and $F(G)=\{a, b, c, d\}$, the vertex-face incident matrix $M_{G}$ is

$$
\left(\begin{array}{llll}
1 & 0 & 1 & 1 \\
1 & 1 & 0 & 1 \\
0 & 1 & 1 & 1 \\
1 & 1 & 1 & 0
\end{array}\right)
$$

LEMMA 2.1. Let $G$ be a triangulation with $n$ vertices and $f$ faces embedded on an orientable surface. Then

$$
M_{G} M_{G}^{T}=D_{G}+2 A_{G}
$$

where $M_{G}^{T}$ is the transpose of $M_{G}$, and $A_{G}$ and $D_{G}$ are the adjacency matrix and the diagonal matrix of vertex degrees of $G$, respectively.

Proof. For any $i, j \in\{1,2, \ldots, n\}$, by the definition of $M_{G}$,

$$
\left(M_{G} M_{G}^{T}\right)_{i i}=\sum_{k=1}^{f} m_{i k} m_{i k}=\sum_{k=1}^{f} m_{i k}=d_{i}
$$


and

$$
\left(M_{G} M_{G}^{T}\right)_{i j}=\sum_{k=1}^{f} m_{i k} m_{j k} .
$$

Note that $m_{i k} m_{j k}=1$ if and only if $v_{i}, v_{j} \in V\left(\phi_{k}\right)$. Since $G$ is a triangulation, the boundary of each face of $G$ is a triangle. So $v_{i}, v_{j} \in V\left(\phi_{k}\right)$ if and only if $\left(v_{i}, v_{j}\right)$ is an edge of $G$. Note that $G$ has no cut edge (since $G$ is a triangulation). Hence, each edge $\left(v_{i}, v_{j}\right)$ is incident to exactly two faces. Thus,

$$
\left(M_{G} M_{G}^{T}\right)_{i j}=\sum_{k=1}^{f} m_{i k} m_{j k}=2 a_{i j}
$$

where $A_{G}=\left(a_{i j}\right)_{n \times n}$.

Hence, we have proved that $M_{G} M_{G}^{T}=D_{G}+2 A_{G}$.

By a suitable labelling of vertices of $G^{\nabla}$ and $G^{\square}$, the adjacency matrices $A\left(G^{\nabla}\right)$ and $A\left(G^{\square}\right)$, and the diagonal matrices of vertex degrees $D\left(G^{\nabla}\right)$ and $D\left(G^{\square}\right)$ of $G^{\nabla}$ and $G^{\square}$ have the following forms:

$$
\begin{array}{cc}
A\left(G^{\nabla}\right)=\left(\begin{array}{cc}
A_{G} & M_{G} \\
M_{G}^{T} & 0_{f}
\end{array}\right), \quad A\left(G^{\square}\right)=\left(\begin{array}{cc}
0_{n} & M_{G} \\
M_{G}^{T} & 0_{f}
\end{array}\right), \\
D\left(G^{\nabla}\right)=\left(\begin{array}{cc}
2 D_{G} & 0_{n \times f} \\
0_{f \times n} & 3 I_{f}
\end{array}\right), \quad D\left(G^{\square}\right)=\left(\begin{array}{cc}
D_{G} & 0_{n \times f} \\
0_{f \times n} & 3 I_{f}
\end{array}\right),
\end{array}
$$

where $A_{G}, D_{G}$, and $M_{G}$ are the adjacency matrix, the diagonal matrix of vertex degrees, and the vertex-face incident matrix of $G$, respectively, and $0_{n \times f}$ and $I_{f}$ are the $n \times f$ matrix with all entries equal to zero and the unit matrix of order $f$. Hence, the Laplacian matrices $L\left(G^{\nabla}\right)$ and $L\left(G^{\square}\right)$ of $G^{\nabla}$ and $G^{\square}$ are

$$
L\left(G^{\nabla}\right)=\left(\begin{array}{cc}
2 D_{G}-A_{G} & -M_{G} \\
-M_{G}^{T} & 3 I_{f}
\end{array}\right), \quad L\left(G^{\square}\right)=\left(\begin{array}{cc}
D_{G} & -M_{G} \\
-M_{G}^{T} & 3 I_{f}
\end{array}\right) .
$$

THEOREM 2.2. Let $G$ be a triangulation with $n$ vertices and $f$ faces embedded on an orientable surface, and $G^{\nabla}$ and $G^{\square}$ the vertex-face graphs of the first kind and the second kind of $G$, respectively. Suppose the normalized Laplacian spectrum of $G$ is $\left\{\theta_{1}, \theta_{2}, \ldots, \theta_{n}\right\}$. Then:

(1) The normalized Laplacian spectrum of $G^{\nabla}$ is $\{\overbrace{1, \ldots, 1}^{f-n}, \frac{9+3 \theta_{i} \pm \sqrt{\left(9+3 \theta_{i}\right)^{2}-120 \theta_{i}}}{12}, i=1,2, \ldots, n\}$.

(2) The normalized Laplacian spectrum of $G^{\square}$ is $\{\overbrace{1, \ldots, 1}^{f-n}, \frac{3 \pm \sqrt{9-6 \theta_{i}}}{3}, i=1,2, \ldots, n\}$.

Proof. (1) Note that the normalized Laplacian characteristic polynomial of $G$ is

$$
\begin{aligned}
\chi(G, x) & =\operatorname{det}\left(x I_{n}-\mathcal{L}_{G}\right) \\
& =\left|x I_{n}-D_{G}{ }^{-1 / 2} L_{G} D_{G}{ }^{-1 / 2}\right| \\
& =\left|(x-1) I_{n}+D_{G}{ }^{-1 / 2} A_{G} D_{G}{ }^{-1 / 2}\right| \\
& =\left|D_{G}{ }^{1 / 2}\right|\left|(x-1) I_{n}+D_{G}^{-1} A_{G}\right|\left|D_{G}{ }^{-1 / 2}\right| \\
& =\left|x I_{n}-\left(I_{n}-D_{G}{ }^{-1} A_{G}\right)\right| .
\end{aligned}
$$


By equations (2.1) and (2.2),

$$
D\left(G^{\nabla}\right)^{-1} A\left(G^{\nabla}\right)=\left(\begin{array}{cc}
\frac{1}{2} D_{G}^{-1} A_{G} & \frac{1}{2} D_{G}{ }^{-1} M_{G} \\
\frac{1}{3} M_{G}{ }^{T} & 0_{f}
\end{array}\right) .
$$

Then

$$
\begin{aligned}
\chi\left(G^{\nabla}, x\right) & =\operatorname{det}\left(x I_{n+f}-\left(I_{n+f}-D\left(G^{\nabla}\right)^{-1} A\left(G^{\nabla}\right)\right)\right) \\
& =\operatorname{det}\left(\begin{array}{cc}
(x-1) I_{n}+\frac{1}{2} D_{G}{ }^{-1} A_{G} & \frac{1}{2} D_{G}{ }^{-1} M_{G} \\
\frac{1}{3} M_{G} & (x-1) I_{f}
\end{array}\right) \\
& =(x-1)^{f} \operatorname{det}\left(\begin{array}{cc}
(x-1) I_{n}+\frac{1}{2} D_{G}{ }^{-1} A_{G} & \frac{1}{2} D_{G}{ }^{-1} M_{G} \\
\frac{1}{3(x-1)} M_{G}^{T} & I_{f}
\end{array}\right) \\
& =(x-1)^{f} \operatorname{det}\left((x-1) I_{n}+\frac{1}{2} D_{G}{ }^{-1} A_{G}-\frac{1}{6(x-1)} D_{G}{ }^{-1} M_{G} M_{G}^{T}\right) .
\end{aligned}
$$

By Lemma 2.1, $M_{G} M_{G}^{T}=D_{G}+2 A_{G}$. Thus,

$$
\begin{aligned}
\chi\left(G^{\nabla}, x\right) & =\left(\frac{1}{6}\right)^{n}(x-1)^{f-n} \operatorname{det}\left(6(x-1)^{2} I_{n}+3(x-1) D_{G}{ }^{-1} A_{G}-D_{G}{ }^{-1}\left(D_{G}+2 A_{G}\right)\right) \\
& =\left(\frac{1}{6}\right)^{n}(x-1)^{f-n} \operatorname{det}\left(\left(6 x^{2}-9 x\right) I_{n}-(3 x-5)\left(I_{n}-D_{G}{ }^{-1} A_{G}\right)\right) .
\end{aligned}
$$

Note that the normalized Laplacian spectrum of $G$ (or $\left.A_{G}\right)$ is $\left\{\theta_{1}, \theta_{2}, \ldots, \theta_{n}\right\}$. Then

$$
\chi\left(G^{\nabla}, x\right)=\left(\frac{1}{6}\right)^{n}(x-1)^{f-n} \prod_{i=1}^{n}\left[6 x^{2}-9 x-(3 x-5) \theta_{i}\right] .
$$

Thus, the zeros of $\chi\left(G^{\nabla}, x\right)$ are

$$
\overbrace{1, \ldots, 1}^{f-n}, \frac{9+3 \theta_{i} \pm \sqrt{\left(9+3 \theta_{i}\right)^{2}-120 \theta_{i}}}{12}, \quad i=1,2, \ldots, n .
$$

(2) Similarly, we can prove that the normalized Laplacian spectrum of $G^{\square}$ is

$$
\{\overbrace{1, \ldots, 1}^{f-n}, \frac{3 \pm \sqrt{9-6 \theta_{i}}}{3}, i=1,2, \ldots, n\}
$$

THEOREM 2.3. Let $G$ be a $k$-regular triangulation with $n$ vertices and $f$ faces embedded on an orientable surface, and $G^{\nabla}$ and $G^{\square}$ the vertex-face graphs of the first kind and the second kind of $G$, respectively. Let $\left\{\lambda_{1}, \lambda_{2}, \ldots, \lambda_{n}\right\}$ be the spectrum of $G$. Then:

(1) The spectrum of $G^{\nabla}$ is $\{\overbrace{0, \ldots, 0}^{f-n}, \frac{\lambda_{i} \pm \sqrt{\lambda_{i}^{2}+8 \lambda_{i}+4 k}}{2}, i=1,2, \ldots, n\}$.

(2) The spectrum of $G^{\square}$ is $\{\overbrace{0, \ldots, 0}^{f-n}, \pm \sqrt{k+2 \lambda_{i}}, i=1,2, \ldots, n\}$.

(3) The Laplacian spectrum of $G^{\nabla}$ is $\{\overbrace{3, \ldots, 3}^{f-n}, \frac{3+2 k-\lambda_{i} \pm \sqrt{\left(3+2 k-\lambda_{i}\right)^{2}-20 k+20 \lambda_{i}}}{2}, i=1,2, \ldots, n\}$.

(4) The Laplacian spectrum of $G^{\square}$ is $\{\overbrace{3, \ldots, 3}^{f-n}, \frac{3+k \pm \sqrt{(3+k)^{2}-8 k+8 \lambda_{i}}}{2}, i=1,2, \ldots, n\}$. 
Proof. (1) By equation (2.1), the characteristic polynomial of $G^{\nabla}$ is

$$
\begin{aligned}
\phi\left(G^{\nabla}, x\right) & =\operatorname{det}\left(\begin{array}{cc}
x I_{n}-A_{G} & -M_{G} \\
-M_{G}^{T} & x I_{f}
\end{array}\right) \\
& =x^{f} \operatorname{det}\left(\begin{array}{cc}
x I_{n}-A_{G} & -M_{G} \\
-\frac{1}{x} M_{G}^{T} & I_{f}
\end{array}\right) \\
& =x^{f} \operatorname{det}\left(x I_{n}-A_{G}-\frac{1}{x} M_{G} M_{G}^{T}\right) .
\end{aligned}
$$

By Lemma 2.1, $M_{G} M_{G}^{T}=D_{G}+2 A_{G}$. Since $G$ is $k$-regular. Thus,

$$
\begin{aligned}
\phi\left(G^{\nabla}, x\right) & =x^{f-n} \operatorname{det}\left(x^{2} I_{n}-x A_{G}-D_{G}-2 A_{G}\right) \\
& =x^{f-n} \operatorname{det}\left(\left(x^{2}-k\right) I_{n}-(x+2) A_{G}\right) .
\end{aligned}
$$

Note that the spectrum of $G$ (or $A_{G}$ ) is $\left\{\lambda_{1}, \lambda_{2}, \ldots, \lambda_{n}\right\}$. Then

$$
\phi\left(G^{\nabla}, x\right)=x^{f-n} \prod_{i=1}^{n}\left[x^{2}-k-(x+2) \lambda_{i}\right] .
$$

Thus, the zeros of $\phi\left(G^{\nabla}, x\right)$ are

$$
\overbrace{0, \ldots, 0}^{f-n}, \frac{\lambda_{i} \pm \sqrt{\lambda_{i}^{2}+8 \lambda_{i}+4 k}}{2}, \quad i=1,2, \ldots, n .
$$

(2) Similarly, by equation (2.1), we can show that the spectrum of $G^{\square}$ is

$$
\{\overbrace{0, \ldots, 0}^{f-n}, \pm \sqrt{k+2 \lambda_{i}}, i=1,2, \ldots, n\} .
$$

(3) By equation (2.3), the Laplacian characteristic polynomial $\mu\left(G^{\nabla}, x\right)$ of $G^{\nabla}$ is

$$
\mu\left(G^{\nabla}, x\right)=\operatorname{det}\left(\begin{array}{cc}
x I_{n}-2 D_{G}+A_{G} & M_{G} \\
M_{G}^{T} & (x-3) I_{f}
\end{array}\right) .
$$

Hence,

$$
\begin{aligned}
\mu\left(G^{\nabla}, x\right) & =(x-3)^{f} \operatorname{det}\left(\begin{array}{cc}
x I_{n}-2 D_{G}+A_{G} & M_{G} \\
\frac{1}{x-3} M_{G}^{T} & I_{f}
\end{array}\right) \\
& =(x-3)^{f} \operatorname{det}\left(x I_{n}-2 D_{G}+A_{G}-\frac{1}{x-3} M_{G} M_{G}^{T}\right) .
\end{aligned}
$$

Since $M_{G} M_{G}^{T}=D_{G}+2 A_{G}$, and $G$ is $k$-regular,

$$
\begin{aligned}
\mu\left(G^{\nabla}, x\right) & =(x-3)^{f-n} \operatorname{det}\left(x(x-3) I_{n}-2(x-3) D_{G}+(x-3) A_{G}-D_{G}-2 A_{G}\right) \\
& =(x-3)^{f-n} \operatorname{det}\left(\left(x^{2}-3 x\right) I_{n}+(5-2 x) k I_{n}+(x-5) A_{G}\right) .
\end{aligned}
$$

Note that the spectrum of $G$ (or $A_{G}$ ) is $\left\{\lambda_{1}, \lambda_{2}, \ldots, \lambda_{n}\right\}$. Thus,

$$
\mu\left(G^{\nabla}, x\right)=(x-3)^{f-n} \prod_{i=0}^{n}\left[x^{2}-3 x+(5-2 x) k+(x-5) \lambda_{i}\right] .
$$


Therefore, the zeros of $\mu\left(G^{\nabla}, x\right)$ are

$$
\overbrace{3, \ldots, 3}^{f-n}, \frac{3+2 k-\lambda_{i} \pm \sqrt{\left(3+2 k-\lambda_{i}\right)^{2}-20 k+20 \lambda_{i}}}{2}, \quad i=1,2, \ldots, n .
$$

(4) Similarly, we can prove that the Laplacian spectrum of $G^{\square}$ is

$$
\{\overbrace{3, \ldots, 3}^{f-n}, \frac{3+k \pm \sqrt{(3+k)^{2}-8 k+8 \lambda_{i}}}{2}, i=1,2, \ldots, n\}
$$

REMARK 2.4. For the $k$-regular triangulations with $n$ vertices and $f$ faces embedded on an orientable surface $\Sigma$ with genus $g$, the three variables $k, g$ and $n$ satisfy the equation $(k-6) n=12(g-1)$, which shows that for fixed genus $g$ not equal to 1 , there are only finitely many such triangulations. Only when the genus is 1 and $k=6$, there are infinite families. For example, for any $m \geq 2, n \geq 2$, the triangular lattices $T^{t}(n, m)$ defined in Section 5 are 6-regular graphs on torus (i.e, genus $g=1$ ).

3. Counting spanning trees of the vertex-face graphs. The enumeration of spanning trees of a graph $G$ was first considered by Kirchhoff in the analysis of electric circuits [12]. It is a problem of fundamental interest in mathematics [1,3,18] and in physics [4, 16, 19]. The number of spanning trees is closely related to the partition function of the $q$-state Potts model in statistical mechanics [9, 20]. Some recent studies on the enumeration of spanning trees and the calculation of their asymptotic growth constants on regular lattices were carried out in $[4,5,15,17]$. We denote by $t(G)$ the number of spanning trees of $G$. For convenience, we present a fundamental result about the normalized Laplacian spectrum and the number of spanning trees $t(G)$ of a graph $G$, for more details see [7].

THEOREM 3.1. Let $G$ be a connected graph with $n$ vertices $m$ edges, then the number of spanning trees of $G$ is

$$
t(G)=\frac{1}{2 m} \prod_{i=1}^{n} d_{i} \prod_{k=2}^{n} \theta_{k}
$$

where $d_{i}$ is the degree of vertex $v_{i}$ of $G$, and the $\theta_{k}$ are the nonzero normalized Laplacian eigenvalue of $G$.

For the notation above we have the following result.

THEOREM 3.2. Let $G$ be a triangulation with $n$ vertices and $f$ faces embedded on an orientable surface $\Sigma$ with genus $g$, and $G^{\nabla}$ and $G^{\square}$ the vertex-face graphs of the first kind and the second kind of $G$, respectively. Then

$$
t\left(G^{\nabla}\right)=3^{n+4 g-3} 5^{n-1} t(G), t\left(G^{\square}\right)=3^{n+4 g-3} 2^{n-1} t(G) .
$$

Proof. Note that $G$ is a triangulation with $n$ vertices and $f$ faces, then $\left|V\left(G^{\nabla}\right)\right|=\left|V\left(G^{\square}\right)\right|=n+f$, $m\left(G^{\nabla}\right)=m+3 f, m\left(G^{\square}\right)=3 f$, and $m$ is the number of edges of $G$. And $3 f=2 m$. Thus,

$$
m\left(G^{\nabla}\right)=3 m, m\left(G^{\square}\right)=2 m
$$

Obviously, the degree sequences of $G^{\nabla}$ and $G^{\square}$ are $\{2 d_{1}, 2 d_{2}, \ldots, 2 d_{n}, \overbrace{3, \ldots, 3}^{f}\}$ and $\{d_{1}, d_{2}, \ldots, d_{n}, \overbrace{3, \ldots, 3}^{f}\}$. 
By Theorem 2.2 and equations (3.1) and (3.2), we have

$$
\begin{aligned}
t\left(G^{\nabla}\right) & =\frac{1}{2 m\left(G^{\nabla}\right)} \prod_{i=1}^{n+f} d_{i}\left(G^{\nabla}\right) \prod_{k=2}^{n+f} \theta_{k}\left(G^{\nabla}\right) \\
& =\frac{2^{n} 3^{f}}{6 m} \prod_{i=1}^{n} d_{i}\left[\frac{3}{2} \times 1^{f-n} \prod_{k=2}^{n}\left(\frac{9+3 \theta_{k}+\sqrt{\left(9+3 \theta_{k}\right)^{2}-120 \theta_{k}}}{12}\right)\left(\frac{9+3 \theta_{k}-\sqrt{\left(9+3 \theta_{k}\right)^{2}-120 \theta_{k}}}{12}\right)\right] \\
& =\frac{3^{f-n+1} 5^{n-1}}{2 m} \prod_{i=1}^{n} d_{i} \prod_{k=2}^{n} \theta_{k} \\
& =3^{f-n+1} 5^{n-1} t(G) .
\end{aligned}
$$

Since $G$ is a triangulation embedded on an orientable surface with genus $g, n-m+f=2-2 g$ and $3 f=2 m$. So $f=2 n+4 g-4$. Hence,

$$
t\left(G^{\nabla}\right)=3^{f-n+1} 5^{n-1} t(G)=3^{n+4 g-3} 5^{n-1} t(G) .
$$

Similarly, we can show that $t\left(G^{\square}\right)=3^{n+4 g-3} 2^{n-1} t(G)$.

By the theorem above, we have:

Corollary 3.3. Let $G$ be a plane triangulation with $n$ vertices. Then

$$
t\left(G^{\nabla}\right)=3^{n-3} 5^{n-1} t(G), t\left(G^{\square}\right)=3^{n-3} 2^{n-1} t(G) .
$$

COROLlary 3.4. Let $G$ be a toroidal triangulation with $n$ vertices. Then

$$
t\left(G^{\nabla}\right)=3^{n+1} 5^{n-1} t(G), t\left(G^{\square}\right)=3^{n+1} 2^{n-1} t(G) .
$$

4. Kirchhoff index and degree-Kirchhoff index of vertex-face graphs. On the basis of electrical network theory, the study of resistance distance was initiated by Klein and Randic [13], and the related index, known as the Kirchoff index, is well studied in [10,21]. For any two vertices $u$ and $v$ in a connected graph $G$, the resistance distance $\Omega(u, v)$ between $u$ and $v$ is defined as the effective resistance between $u$ and $v$ by replacing each edge of $G$ with unit resistors. The Kirchhoff index of a graph $G$, denoted by $\operatorname{Kf}(G)$, is defined as the sum of resistance distances between all pairs of vertices [2, 13]; that is,

$$
\operatorname{Kf}(G)=\sum_{\{u, v\} \subset V(G)} \Omega(u, v) .
$$

At almost the same time, Gutman and Mohar [10] and Zhu et al. [23] proved that, if $G$ is a connected simple graph with $n$ vertices, then

$$
\mathrm{Kf}(G)=n \sum_{i=1}^{n-1} \frac{1}{\mu_{i}}
$$

where $\mu_{1}, \mu_{2}, \ldots, \mu_{n-1}$ are the non-zero Laplacian eigenvalues of $G$.

Recently, one modification of the Kirchhoff index, which takes the degrees of the graph into account, has been considered. The degree-Kirchhoff index is defined by Chen and Zhang [6]:

$$
\mathrm{Kf}^{\prime}(G)=\sum_{\{u, v\} \subset V(G)} d_{u} d_{v} \Omega(u, v)
$$


Electronic Journal of Linear Algebra, ISSN 1081-3810

A publication of the International Linear Algebra Society

Volume 36, pp. 616-628, September 2020 .

623

On the Vertex-Face Graphs of Triangulations

where $d_{u}$ is the degree of the vertex $u$ of $G$. They proved that, if $G$ is a connected simple graph with $n$ vertices $m$ edges, then

$$
\mathrm{Kf}^{\prime}(G)=2 m \sum_{k=2}^{n} \frac{1}{\theta_{k}(G)}
$$

where $\theta_{2}, \theta_{3}, \ldots, \theta_{n}$ are the non-zero normalized Laplacian eigenvalues of $G$.

THEOREM 4.1. Let $G$ be a triangulation with $n$ vertices and $f$ faces embedded on an orientable surface, and $G^{\nabla}$ and $G^{\square}$ the vertex-face graphs of the first kind and the second kind of $G$, respectively. Then

$$
\mathrm{Kf}^{\prime}\left(G^{\nabla}\right)=\frac{3 f}{5}(15 f-6 n+1)+\frac{27}{5} \mathrm{Kf}^{\prime}(G)
$$

and

$$
\mathrm{Kf}^{\prime}\left(G^{\square}\right)=3 f(2 f-2 n+1)+6 \mathrm{Kf}^{\prime}(G) .
$$

Proof. Note that $G$ is a triangulation with $n$ vertices and $f$ faces, then $\left|V\left(G^{\nabla}\right)\right|=\left|V\left(G^{\square}\right)\right|=n+f$, $m\left(G^{\nabla}\right)=m+3 f, m\left(G^{\square}\right)=3 f$, where $m$ is the number of edges of $G$. And $3 f=2 m$, then $m\left(G^{\nabla}\right)=$ $3 m, m\left(G^{\square}\right)=2 m$.

By Theorem 2.2 and equation (4.2), the degree-Kirchhoff index of $G^{\nabla}$ is

$$
\begin{aligned}
\operatorname{Kf}^{\prime}\left(G^{\nabla}\right) & =2 m\left(G^{\nabla}\right) \sum_{k=2}^{n+f} \frac{1}{\theta_{k}\left(G^{\nabla}\right)} \\
& =6 m\left((f-n)+\frac{1}{\frac{3}{2}}+\sum_{k=2}^{n}\left(\frac{12}{9+3 \theta_{k}+\sqrt{\left(9+3 \theta_{k}\right)^{2}-120 \theta_{k}}}+\frac{12}{9+3 \theta_{k}-\sqrt{\left(9+3 \theta_{k}\right)^{2}-120 \theta_{k}}}\right)\right) \\
& =6 m(f-n)+4 m+6 m \sum_{k=2}^{n}\left(\frac{9}{5 \theta_{k}}+\frac{3}{5}\right) \\
& =\frac{3 f}{5}(15 f-6 n+1)+\frac{27}{5} \operatorname{Kf}^{\prime}(G)
\end{aligned}
$$

and the degree-Kirchhoff index of $G^{\square}$ is

$$
\begin{aligned}
\operatorname{Kf}^{\prime}\left(G^{\square}\right) & =2 m\left(G^{\square}\right) \sum_{k=2}^{n+f} \frac{1}{\theta_{k}\left(G^{\square}\right)} \\
& =4 m\left((f-n)+\frac{1}{2}+\sum_{k=2}^{n}\left(\frac{3}{3+\sqrt{9-6 \theta_{k}}}+\frac{3}{3-\sqrt{9-6 \theta_{k}}}\right)\right) \\
& =4 m(f-n)+2 m+4 m \sum_{k=2}^{n} \frac{3}{\theta_{k}} \\
& =3 f(2 f-2 n+1)+6 \operatorname{Kf}^{\prime}(G) .
\end{aligned}
$$

THEOREM 4.2. Let $G$ be a $k$-regular triangulation with $n$ vertices and $f$ faces embedded on an orientable surface, and $G^{\nabla}$ and $G^{\square}$ the vertex-face graphs of the first kind and the second kind of $G$, respectively. Then

$$
\operatorname{Kf}\left(G^{\nabla}\right)=\frac{f^{2}-n^{2}}{3}+\frac{n+f}{k+3}+\frac{(n+f)(n-1)}{5}+\frac{(n+f)(k+3)}{5 n} \operatorname{Kf}(G)
$$

and

$$
\mathrm{Kf}\left(G^{\square}\right)=\frac{f^{2}-n^{2}}{3}+\frac{n+f}{k+3}+\frac{(n+f)(k+3)}{2 n} \operatorname{Kf}(G) .
$$


Proof. Suppose that the spectrum of $G$ is $\left\{\lambda_{1}, \lambda_{2}, \ldots, \lambda_{n}=k\right\}$. Then the Laplacian spectrum of $G$ is $\left\{\mu_{1}=k-\lambda_{1}, \mu_{2}=k-\lambda_{2}, \ldots, \mu_{n-1}=k-\lambda_{n-1}, \mu_{n}=0\right\}$. By equation (4.1), the Kirchhoff index of $G$ is

$$
\mathrm{Kf}(G)=n \sum_{i=1}^{n-1} \frac{1}{\mu_{i}(G)}=n \sum_{i=1}^{n-1} \frac{1}{k-\lambda_{i}} .
$$

Note that $V\left(G^{\nabla}\right)=V\left(G^{\square}\right)=n+f$. By Theorem 2.3, the Kirchhoff index of $G^{\nabla}$ is

$$
\begin{aligned}
\mathrm{Kf}\left(G^{\nabla}\right)= & (n+f)\left[\frac{1}{3}(f-n)+\frac{1}{k+3}+\sum_{i=1}^{n-1} \frac{1}{\mu_{i}\left(G^{\nabla}\right)}\right] \\
= & \frac{1}{3}\left(f^{2}-n^{2}\right)+\frac{n+f}{k+3}+(n+f) \sum_{i=1}^{n-1}\left(\frac{2}{3+2 k-\lambda_{i}+\sqrt{\left(3+2 k-\lambda_{i}\right)^{2}-20 k+20 \lambda_{i}}}\right. \\
& \left.+\frac{2}{3+2 k-\lambda_{i}-\sqrt{\left(3+2 k-\lambda_{i}\right)^{2}-20 k+20 \lambda_{i}}}\right) \\
= & \frac{f^{2}-n^{2}}{3}+\frac{n+f}{k+3}+\frac{(n+f)(n-1)}{5}+\frac{(n+f)(k+3)}{5 n} \operatorname{Kf}(G),
\end{aligned}
$$

and the Kirchhoff index of $G^{\square}$ is

$$
\begin{aligned}
\operatorname{Kf}\left(G^{\square}\right) & =(n+f)\left[\frac{1}{3}(f-n)+\frac{1}{k+3}+\sum_{i=1}^{n-1} \frac{1}{\mu_{i}(G \square)}\right] \\
& =\frac{1}{3}\left(f^{2}-n^{2}\right)+\frac{n+f}{k+3}+(n+f) \sum_{i=1}^{n-1}\left(\frac{2}{3+k+\sqrt{(3+k)^{2}-8 k+8 \lambda_{i}}}+\frac{2}{3+k-\sqrt{(3+k)^{2}-8 k+8 \lambda_{i}}}\right) \\
& =\frac{f^{2}-n^{2}}{3}+\frac{n+f}{k+3}+\frac{(n+f)(k+3)}{2 n} \operatorname{Kf}(G) .
\end{aligned}
$$

5. Applications. As applications of the results in Section 2, 3 and 4, in this section we enumerate spanning trees of the dual lattice of the 3.12.12 lattice and the dice lattice with toroidal boundary condition in the context of statistical physics. We also obtain a formula of Kirchhoff indices of these two lattices.

The 3.12.12 lattice $R^{t}(n, m)$ with toroidal boundary condition is shown in Figure 2(a). For any given vertex, the incident faces are list in, say, clockwise order, by the number of edges in the face, the list being the name of the lattice. Several lattices get multiple names, depending on the starting face, the convention is then to choose the lexicographically smallest name [14]. This is the origin of the name of the 3.12 .12 lattice. For $R^{t}(n, m)$ in Figure 2(a), all $a_{i}$ 's, $a_{i}^{*}$ 's, $b_{i}$ 's, and $b_{i}^{*}$ 's are some vertices on the left, right, lower and upper boundaries, respectively, and the left and right (resp. the lower and upper) boundaries of the picture are identified such that $\left(a_{1}, a_{1}^{*}\right),\left(a_{2}, a_{2}^{*}\right), \ldots,\left(a_{m}, a_{m}^{*}\right)$ and $\left(b_{1}, b_{1}^{*}\right),\left(b_{2}, b_{2}^{*}\right), \ldots,\left(b_{n}, b_{n}^{*}\right)$ are edges in $R^{t}(n, m)$. The 3.12.12 lattice $R^{t}(n, m)$ has been used by Fisher [8] in a dimer formulation of the Ising model.

The triangular lattice with toroidal boundary condition, denoted by $T^{t}(n, m)$, can be regarded as an $n \times m$ square lattice with toroidal boundary condition with an additional diagonal edge added, in the same way, to every square, see Figure 2(b).

The dice lattice $D^{t}(n, m)$ with toroidal boundary condition is the dual lattice of the kagomé lattice, depicted in Figure 2(c), which can be obtained from the hexagonal toroidal lattice by inserting a new vertex $v_{f}$ to each face $f$ and adding three edges $\left(v_{f}, u_{i}(f)\right)$, where $u_{1}(f), u_{2}(f)$ and $u_{3}(f)$ are mutually non-adjacent vertices on the boundary of $f$, such that two vertices $v_{f_{1}}$ and $v_{f_{2}}$ have a common neighbor $u_{i}(f), i \in\{1,2,3\}$, where $f_{1}, f_{2}$ are two adjacent faces. 


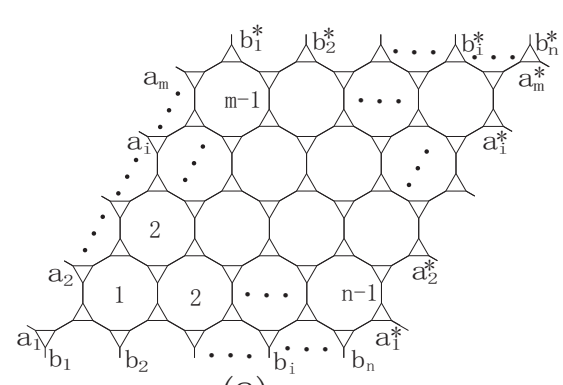

(a)

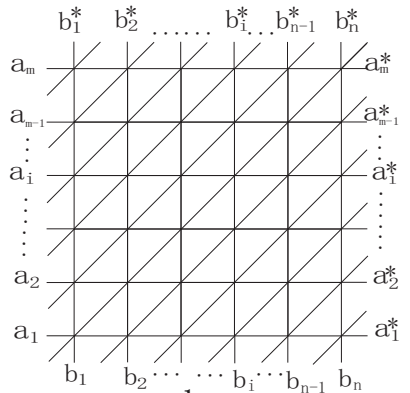

(b)

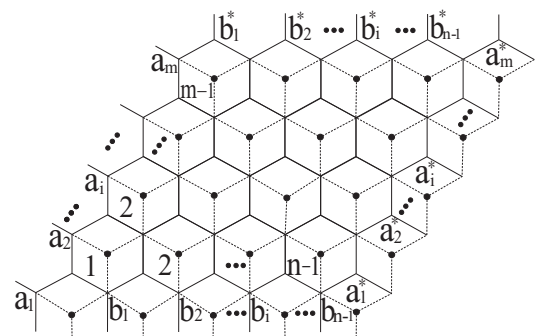

(C)

FIGURE 2. (a) The 3.12 .12 lattice $R^{t}(n, m)$ with toroidal boundary condition. (b) The triangular lattice $T^{t}(n, m)$ with toroidal boundary condition. (c) The dice lattice $D^{t}(n, m)$ with toroidal boundary condition.

It is not difficult to see that the dual lattice of 3.12.12 lattice $R^{t}(n, m)$ and the dice lattice $D^{t}(n, m)$ are the two vertex-face graphs of the triangular lattice $T^{t}(n, m)$, that is, $R^{t}(n, m)^{\perp}=T^{t}(n, m)^{\nabla}, D^{t}(n, m)=$ $T^{t}(n, m)^{\square}$. Note that the eigenvalues of $T^{t}(n, m)[22]$ are

$$
\lambda_{i j}=2 \cos \frac{2 \pi i}{n}+2 \cos \frac{2 \pi j}{m}+2 \cos \left(\frac{2 \pi i}{n}+\frac{2 \pi j}{m}\right), \quad 0 \leq i \leq n-1, \quad 0 \leq j \leq m-1 .
$$

Then, by Theorem 2.3 , the spectra of $R^{t}(n, m)^{\perp}$ and $D^{t}(n, m)$ are

$$
\left\{0^{m n}, \frac{\lambda_{i j} \pm \sqrt{\lambda_{i j}^{2}+8 \lambda_{i j}+24}}{2}, 0 \leq i \leq n-1,0 \leq j \leq m-1\right\}
$$

and

$$
\left\{0^{m n}, \pm \sqrt{2\left(3+2 \cos \frac{2 \pi i}{n}+2 \cos \frac{2 \pi j}{m}+2 \cos \left(\frac{2 \pi i}{n}+\frac{2 \pi j}{m}\right)\right)}, 0 \leq i \leq n-1,0 \leq j \leq m-1\right\}
$$

respectively. The Laplacian spectra of $R^{t}(n, m)^{\perp}$ and $D^{t}(n, m)$ are

$$
\left\{3^{m n}, \frac{15-\lambda_{i j} \pm \sqrt{\lambda_{i j}^{2}-10 \lambda_{i j}+105}}{2}, 0 \leq i \leq n-1,0 \leq j \leq m-1\right\}
$$

and

$$
\left\{3^{m n}, \frac{9 \pm \sqrt{9+8\left(3+2 \cos \frac{2 \pi i}{n}+2 \cos \frac{2 \pi j}{m}+2 \cos \left(\frac{2 \pi i}{n}+\frac{2 \pi j}{m}\right)\right)}}{2}, 0 \leq i \leq n-1,0 \leq j \leq m-1\right\} .
$$

Shrock and $\mathrm{Wu}[15]$ showed that the number of spanning trees and the spanning tree entropy of $R^{t}(n, m)$ can be expressed as

$$
t\left(R^{t}(n, m)\right)=\frac{75}{m n} \prod_{\substack{i=0 \\(i, j) \neq(0,0)}}^{n-1} \prod_{j=0}^{m-1} 30\left[3-\cos \frac{2 \pi i}{m}-\cos \frac{2 \pi j}{n}-\cos \left(\frac{2 \pi i}{m}+\frac{2 \pi j}{n}\right)\right]
$$

and

$$
\lim _{n, m \rightarrow \infty} \frac{1}{6 m n} \log \left[t\left(R^{t}(n, m)\right)\right]
$$




$$
=\frac{1}{6} \log 30+\frac{1}{24 \pi^{2}} \int_{0}^{2 \pi} \int_{0}^{2 \pi} \log [6-2 \cos x-2 \cos y-2 \cos (x+y)] d x d y \approx 0.7206,
$$

and the number of spanning trees and the spanning tree entropy of $T^{t}(n, m)$ can be expressed as

$$
\begin{gathered}
t\left(T^{t}(n, m)\right)=\frac{1}{m n} \prod_{\substack{i=0 \\
(i, j) \neq(0,0)}}^{n-1} \prod_{j=0}^{m-1}\left[6-2 \cos \frac{2 \pi i}{m}-2 \cos \frac{2 \pi j}{n}-2 \cos \left(\frac{2 \pi i}{m}+\frac{2 \pi j}{n}\right)\right], \\
\lim _{n, m \rightarrow \infty} \frac{\log \left[t\left(T^{t}(n, m)\right)\right]}{n m}=\frac{1}{4 \pi^{2}} \int_{0}^{2 \pi} \int_{0}^{2 \pi} \log [6-2 \cos x-2 \cos y-2 \cos (x+y)] d x d y \approx 1.6153 .
\end{gathered}
$$

Glasser and $\mathrm{Wu}$ [11] also derived the result above for the spanning tree entropy of the triangular lattice with toroidal boundary.

Since $R^{t}(n, m)^{\perp}=T^{t}(n, m)^{\nabla}, D^{t}(n, m)=T^{t}(n, m)^{\square}$. By Corollary 3.4 and equation (5.3), the following results are immediate.

TheOREM 5.1. Let $R^{t}(n, m)$ be the 3.12.12 lattice with toroidal boundary condition. Then

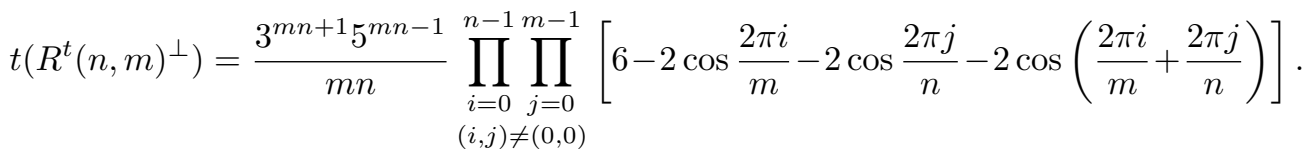

THEOREM 5.2. Let $D^{t}(n, m)$ be the dice lattice with toroidal boundary condition. Then

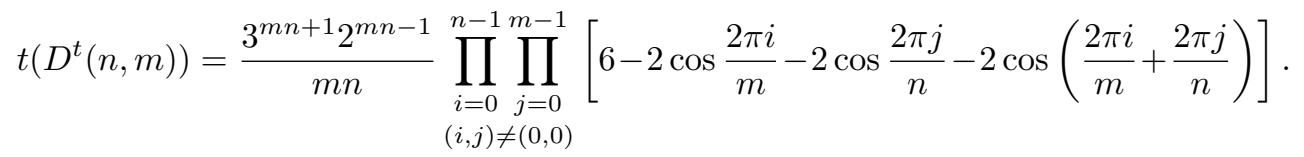

By equation (5.2), a direct result of Theorem 5.1 is the following corollary.

COROLlary 5.3. Let $R^{t}(n, m)$ be the 3.12.12 lattice with toroidal boundary condition. Then

$$
3 t\left(R^{t}(n, m)\right)=25 t\left(R^{t}(n, m)^{\perp}\right)
$$

REMARK 5.4. It is well known that a connected plane graph $G$ and its dual graph $G^{\perp}$ have the same number of spanning trees. The corollary above implies that this is not true for graphs embedded on the torus.

Note that the triangular lattice with toroidal boundary condition $T^{t}(n, m)$ is a 6-regular graph, which has $m n$ vertices and $2 m n$ faces. By equation (5.1), the Kirchoff index of $T^{t}(n, m)$ is

$$
\mathrm{Kf}\left(T^{t}(n, m)\right)=\frac{m n}{2} \sum_{\substack{0 \leq i \leq n-1 \\ 0 \leq j \leq m-1 \\(i, j) \neq(0,0)}} \frac{1}{3-\cos \frac{2 \pi i}{n}-\cos \frac{2 \pi j}{m}-\cos \left(\frac{2 \pi i}{n}+\frac{2 \pi j}{m}\right)}
$$

Then, by equations (4.3), (4.4) and (5.4), the following results are immediate. 
TheOREm 5.5. Let $R^{t}(n, m)$ be the 3.12.12 lattice with toroidal boundary condition. Then the Kirchhoff index of $R^{t}(n, m)^{\perp}$ is

$$
\mathrm{Kf}\left(R^{t}(n, m)^{\perp}\right)=\frac{8 m^{2} n^{2}}{5}-\frac{4 m n}{15}+\frac{27 m n}{10} \sum_{\substack{0 \leq i \leq n-1 \\ 0 \leq j \leq m-1 \\(i, j) \neq(0,0)}} \frac{1}{3-\cos \frac{2 \pi i}{n}-\cos \frac{2 \pi j}{m}-\cos \left(\frac{2 \pi i}{n}+\frac{2 \pi j}{m}\right)} .
$$

Theorem 5.6. Let $D^{t}(n, m)$ be the dice lattice with toroidal boundary condition. Then the Kirchhoff index of $D^{t}(n, m)$ is

$$
\mathrm{Kf}(D L(m, n))=m^{2} n^{2}+\frac{m n}{3}+\frac{27 m n}{4} \sum_{\substack{0 \leq i \leq n-1 \\ 0 \leq j \leq m-1 \\(i, j) \neq(0,0)}} \frac{1}{3-\cos \frac{2 \pi i}{n}-\cos \frac{2 \pi j}{m}-\cos \left(\frac{2 \pi i}{n}+\frac{2 \pi j}{m}\right)} .
$$

Acknowledgment. The authors are grateful to the anonymous referee and the editor for their careful reading and valuable comments which result in an improvement of the original manuscript. This work was supported by the National Natural Science Foundation of China (no. 11571139 and no. 11701324) and the Program for Outstanding Young Scientific Research Talents in Fujian Province University.

\section{REFERENCES}

[1] N.L. Biggs. Algebraic Graph Theory, second edition. Cambridge University Press, Cambridge, 1993.

[2] D. Bonchev, A.T. Balaban, X. Liu, and D.J. Klein. Molecular cyclicity and centricity of polycyclic graph: I. Cyclicty based on resistance distances or reciprocal distances. Int. J. Quantum Chem., 50:1-20, 1994.

[3] R. Burton and R. Pemantle. Local characteristics, entropy and limit theorems for spanning trees and domino tilings via transfer-impedances. Ann. Probab., 21:1329-1371, 1993.

[4] S.-C. Chang and R. Shrock. Some exact results for spanning trees on lattices. J. Phys. A: Math. Gen., 39:5653-5658, 2006.

[5] S.-C. Chang and W. Wang. Spanning trees on lattices and integral identities. J. Phys. A: Math. Gen., 39:10263-10275, 2006.

[6] H.Y. Chen and F.J. Zhang. Resistance distance and the normalized Laplacian spectrum. Discrete Math., 155:654-661, 2007.

[7] F.R.K. Chung. Spectral Graph Theory. American Mathematical Society, Providence, 1997.

[8] M.E. Fisher. On the dimer solution of planar Ising models. J. Math. Phys., 7:1776-1781, 1996.

[9] C.M. Fortuin and P.W. Kasteleyn. On the random cluster model. I. Introduction and relation to other models. Physica, $57: 536-564,1972$.

[10] I. Gutman and B. Mohar. The quasi-Wiener and the Kirchhoff indices coincide. J. Chem. Inf. Comput. Sci., 36:982-985, 1996.

[11] M.L. Glasser and F.-Y. Wu. On the entropy of spanning trees on a large triangular lattice. The Ramanujian Journal, 10:205-214, 2005.

[12] G. Kirchhoff. Über die Auflösung der Gleichungen, auf welche man bei der Untersuchung der linearen Verteilung galvanischer Ströme geführt wird. Ann. Phys. Chem., 72:497-508, 1847.

[13] D.J. Klein and M. Randić. Resistance distance. J. Math. Chem., 12:81-95, 1993.

[14] R. Parviainen. Connectivity Properties of Archimedean and Laves Lattices. Doctoral dissertation, Department of Mathematics, Uppsala University, 2004.

[15] R. Shrock and F.-Y. Wu. Spanning trees on graphs and lattices in d dimensions. J. Phys. A: Math. Gen., 33:3881-3902, 2000 .

[16] H.N.V. Temperley. The enumeration of graphs on large periodic lattices. In: D.J.A. Welsh and D.R. Woodall (editors), Combinatorics: Proc. Combinatorial Mathematics, The Institute of Mathematics and its Applications, Oxford, 356357, 1972.

[17] W.-J. Tzeng and F.-Y.Wu. Spanning trees on hypercubic lattices and nonorientable surfaces. Appl. Math. Lett., 13:19-25, 2000. 
Electronic Journal of Linear Algebra, ISSN 1081-3810

A publication of the International Linear Algebra Society

Volume 36, pp. 616-628, September 2020 .

[18] D.J.A. Welsh. Complexity: Knots, Colourings, and Counting. London Math. Soc. Lecture Notes Series, Vol. 186, Cambridge University Press, Cambridge, 1993.

[19] F.-Y. Wu. Number of spanning trees on a lattice. J. Phys. A: Math. Gen., 10:L113-L115, 1977.

[20] F.-Y. Wu. The Potts model. Rev. Mod. Phys., 54:235-268, 1982.

[21] J.W. Xiao and I. Gutman. Resistance distance and Laplacian spectrum. Theor. Chem. Acc., 110:284-289, 2003.

[22] W.G. Yan and Z.H. Zhang. Asymptotic energy of lattices. Physica A, 388:1463-1471, 2009.

[23] H.Y. Zhu, D.J. Klein, and I. Lakovits. Extensions of the Wiener number. J. Chem. Inf. Comput. Sci., 36:420-428, 1996. 